\title{
2nd Conference on Photoacoustics and Photothermal Theory and Applications (CPPTA)
}

\author{
Tomasz Starecki ${ }^{1}$
}

Published online: 5 November 2015

(C) The Author(s) 2015. This article is published with open access at Springerlink.com

This special issue contains selected papers presented during the 2nd Conference on Photoacoustics and Photothermal Theory and Applications (CPPTA), which was held in Warsaw, Poland, from 23 to 26 of September 2014. The conference was organized by the Faculty of Electronics and Information Technology, Warsaw University of Technology.

Photoacoustic and photothermal techniques have been already implemented in many applications and are used as practical and unique measuring tools. On the other hand, the methods are still being developed, which results in extending the range of their potential applications and constant improvement of specifications of such instrumentation. The Conference on Photoacoustic and Photothermal Theory and Applications has been planned to be a forum where scientists and engineers who deal with photoacoustic or photothermal techniques can share their experiences and ideas. A goal was to make the conference a regular event attended not only by top world scientists but also available for the young people, in particular, graduate and Ph.D. students, who have the opportunity to present the results of their research, but most of all, to learn about the latest discoveries and trends, meet the world's experts in the photoacoustic and photothermal fields, and listen to speeches, during which top international scientists summarize their experiences and talk about new ideas and perspectives of further development in the photoacoustic and photothermal fields. During the second CPPTA, such talks were given by the following professors: Danièle Fournier, Frank Tittel, Andreas Mandelis, András Miklós, and Juan José Alvarado-Gil.

Tomasz Starecki

tomasz@starecki.com

1 Faculty of Electronics and Information Technology, Warsaw University of Technology, Nowowiejska 15/19, 00-665 Warsaw, Poland 
Scope of the conference covers virtually all the aspects of photoacoustic, photothermal, and related research, i.e., theory, instrumentation, and a wide range of applications, including but not limited to

- theoretical considerations,

- instrumentation and methodology,

- spectroscopy, applications in chemistry,

- sensors, actuators, and industrial applications (including environmental sensors, generation of ultrasound, etc.),

- applications in medicine, biology, and agriculture,

- imaging (including thermography, tomography, microscopy, depth profiling, etc.),

- ultrafast, micro/nanoscale, and nonlinear phenomena,

- thermophysics (including characterization of materials), and

- other aspects and applications of photoacoustic and photothermal techniques.

All the abstracts submitted for the 2nd CPPTA were carefully reviewed, and finally over 60 works by over 170 authors were accepted for oral and poster presentations. All papers published in this special issue have undergone the standard review process of the International Journal of Thermophysics. I would like to thank all the reviewers for their valuable comments and suggestions, which substantially improved the scientific level of the submitted papers. I mention my special thanks to Dr. William M. Haynes, Editorin-Chief of the International Journal of Thermophysics, for his large contribution towards the publication of this issue. His kindness and assistance are difficult to be overestimated. I greatly appreciate the support and valuable advice that I have received from the members of our scientific committee and participants of the conference. Finally, I would like to invite all our former participants as well as those who have not yet attended our conferences to the third CPPTA, which will be held in September 2016 in Warsaw.

Open Access This article is distributed under the terms of the Creative Commons Attribution 4.0 International License (http://creativecommons.org/licenses/by/4.0/), which permits unrestricted use, distribution, and reproduction in any medium, provided you give appropriate credit to the original author(s) and the source, provide a link to the Creative Commons license, and indicate if changes were made. 